\title{
New Trajectory Control Directional MWD Accuracy Prediction and Wellbore Positioning Method
}

\author{
${ }^{1,2}$ Ahmed Abd Elaziz Ibrahim, ${ }^{2,3}$ Tagwa Ahmed Musa and ${ }^{1}$ Tang FengLin ${ }^{1}$ Yao AiGou \\ ${ }^{1}$ Engineering Faculty, China University of Geosciences, Wuhan-430074, China \\ ${ }^{2}$ Engineering Faculty, Sudan University of Science and Technology, Sudan \\ ${ }^{3}$ Earth Resources Faculty, China University of Geosciences, Wuhan-430074, China
}

\begin{abstract}
The deviation control is to restrict the drilling direction of the bit from time to time. The drilling direction is of course depending on the direction of the resultant forces acting on the bit. What is the relationship between these directions? Are there any other influential factors? Answers to such questions, different points of view were subjected to analysis.
\end{abstract}

Key words: Wellbore Trajectory, Bit Trajectory, Actual/Planned Path, Measurement While Drilling (MWD), Logging While Drilling (LWD), Position Uncertainty, Error Accuracy Prediction, Weighting Function

\section{INTRODUCTION}

In the rectangular coordinate system shown in Fig. 1, the side forces $R_{P}$ and $R_{Q}$ are acting along $X$-axis and $\mathrm{Y}$-axis respectively. The resultant force $\mathrm{R}$ is combined by three mutually perpendicular components; they are $\mathrm{R}_{\mathrm{P}}, \mathrm{R}_{\mathrm{Q}}$ and the weight on bit $\mathrm{P}_{\mathrm{B}}$.

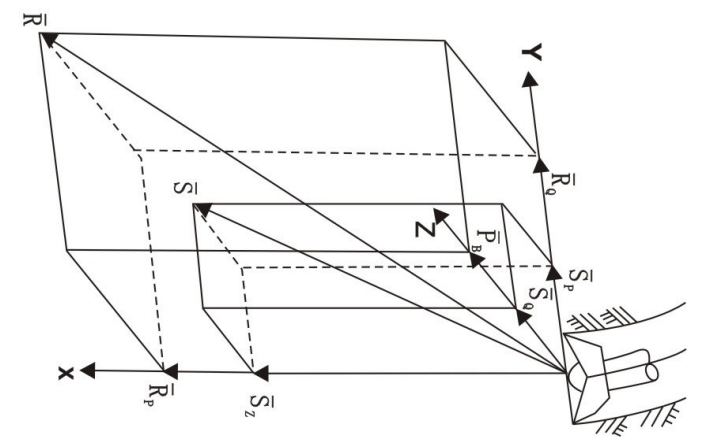

Fig. 1: 3D Relationship between Forces and Displacements

$\overline{S_{Z}}$ is the axial penetration due to $\mathrm{P}_{\mathrm{B}}$ in time interval $\Delta \mathrm{t}$. $\overline{S_{P}}$ is the side cutting in $\mathrm{Y}$-axis due to $\mathrm{R}_{\mathrm{P}}$ in $\Delta \mathrm{t} . \overline{S_{Q}}$ is the side cutting in $\mathrm{Z}$-axis due to $\mathrm{R}_{\mathrm{Q}}$ in $\Delta$ t. It is clear that the drilling direction would not be the same as that of the resultant force and the magnitudes of planned/actual path depends on many influential factors, such as rock properties, formation characteristics, types of bit, etc.

Hole Deviation Mathematical Definition: The wellbore trajectory is defined as a series of surveyed points in 3D space. These points along the planned path are called the Measured Depth (MD*), associated with $M D^{*}$ is north $\left(\mathrm{N}^{*}\right)$, east $\left(\mathrm{E}^{*}\right)$, Total Vertical Depth
(TVD*), Inclination $\left(I^{*}\right)$ and azimuth $\left(\mathrm{A}^{*}\right)$, respectively, planned values North, East, True Vertical Depth, Inclination and Azimuth. These points are jointed together to form a continuous trajectory with a geometric calculations method. Eight components collectively define hole deviation control; they are based on lineal and angular differences between the actual and planned well paths.

$$
\begin{aligned}
& \mathrm{V}=\cos \left(\mathrm{I}_{\mathrm{P}}\right) \cos \left(\mathrm{A}_{\mathrm{P}}\right)\left(\mathrm{N}_{\mathrm{b}}-\mathrm{N}^{*}\right)+\sin \left(\mathrm{I}_{\mathrm{p}}\right) \cos \left(\mathrm{A}_{\mathrm{P}}\right)\left(\mathrm{E}_{\mathrm{b}}-\mathrm{E}^{*}\right)- \\
& \quad \sin \left(\mathrm{A}_{\mathrm{P}}\right)\left(\mathrm{TVD}_{\mathrm{b}}-\mathrm{TVD}^{*}\right) \\
& \mathrm{H}=\cos \left(\mathrm{I}_{\mathrm{P}}\right)\left(\mathrm{E}_{\mathrm{b}}-\mathrm{E}^{*}\right)-\sin \left(\mathrm{I}_{\mathrm{P}}\right)\left(\mathrm{N}_{\mathrm{b}}-\mathrm{N}^{*}\right) \\
& \mathrm{A}=\mathrm{A}_{\mathrm{b}}-\mathrm{A}^{*} \quad \mathrm{I}=\mathrm{I}_{\mathrm{b}}-\mathrm{I}^{*} \\
& \Delta \mathrm{V}_{\mathrm{r}}^{\mathrm{n}}=100 \frac{V^{n}-V^{n+1}}{\Delta L^{n}} \quad \Delta H_{r}^{n}=100 \frac{H^{n}-H^{n-1}}{\Delta L^{n}} \\
& \left(A_{V}\right)_{r}^{n}=\Delta \Delta \varphi_{\mathrm{r}}^{\mathrm{n}}=100 \frac{\Delta A^{n}-\Delta A^{n-1}}{\Delta L^{n}} \\
& \left(I_{H}\right)_{r}^{n}=\Delta \Delta \theta_{r}^{n}=100 \frac{\Delta I^{n}-\Delta I^{n+1}}{\Delta L^{n}}
\end{aligned}
$$

The superscript (n) in the definitions of each relative change is refer to the respective values during the prior computing of hole deviation; (n-1) refers to values at planned hole drilled between the two foregoing hole deviation computations. The superscript $\left(^{*}\right)$ defines the measured data and the subscript (b) refers to current well bore total depth. Thus $\Delta \mathrm{L}$ is $\left(M D^{*}\right)^{(n)}$ which is preferably somewhat short. Performing two successive coordinate axis rotations derive the equations for $(\mathrm{V})$ and $(\mathrm{H})$ the first rotation is by the deviation angle $\theta^{*}$ about the TVD axis. The aforementioned vector is orthogonal to the planned path at $\mathrm{MD}^{*}$, then the required $\triangle T V D$ " equals zero; i.e. Respective to hole deviation, a preferable method by which to 
mathematically represent the entire planned drill path is to parametrically define each Cartesian coordinate and hole inclination and azimuth, in terms of measured depth. That is the planned path is designed and then represented as follows:

$\mathrm{N}_{\mathrm{MD}}=\mathrm{P}_{1(\mathrm{MD})} ; \quad \mathrm{E}_{\mathrm{MD}}=\mathrm{P}_{2(\mathrm{MD}) ;} \quad \mathrm{TVD}_{\mathrm{MD}}=\mathrm{P}_{3(\mathrm{MD})} ; \quad \Phi_{\mathrm{MD}}$ $=\mathrm{P}_{4(\mathrm{MD})} ; \quad \Theta_{\mathrm{MD}}=\mathrm{P}_{5(\mathrm{MD})}$

The rate of change in lineal relationship between the planned and actual well paths is assumed to remain the same over small distances; this assumption is often completely valid. As the hole is drilled, it is necessary to determine where on the plan one would prefer the wellbore to exist. The linear distance between the current bottom hole location and a point on the planned path is computed with the $3 \mathrm{D}$ distance formulas. This is generally represented by Eq. 1

$D_{3 D}\left(N_{b}, E_{h}, T V D_{h}, M D\right)=$

$\sqrt{\left[\left(N_{h}-N_{M D}\right)^{2}+\left(E_{h}-E_{M D}\right)^{2}\right]+\left(T V D_{h}-T V D_{M D}\right)^{2}}$

Let $\mathrm{MD}^{*}$ represent the measured depth along the planned path, whose respective Cartesian coordinates minimize the distance computed with Eq.1. Therefore, $\mathrm{MD}^{*}$ found by taking the derivative of Eq.1 with respect to MD and setting the result equal zero.

$\frac{d D_{3 D}}{d M D}=\frac{\left(N_{M D}-N_{b}\right) \frac{d N_{M D}}{d M D}+\left(E_{M D}-E_{b}\right) \frac{d E_{M D}}{d M D}+\left(T V D_{M D}-T V D_{b}\right) \frac{d T V D_{M D}}{d M D}}{\sqrt{\left(N_{b}+N_{M D}\right)^{2}+\left(E_{b}-E_{M D}\right)^{2}+\left(T V D_{b}-T V D_{M D}\right)^{2}}}$

The measured depth that sets the right hand side of Eq. 2 equal zero is MD*; therefore, the denominator may be ignored and MD* is found by solving Eq. 2 .

$d D_{3 D}=\left(N_{M D}-N_{b}\right) \frac{d N_{M D}}{d M D}+\left(E_{M D}-E_{b}\right) \frac{d E_{M D}}{d M D}$

$+\left(T V D_{M D}-T V D_{b}\right) \frac{d T V D_{M D}}{d M D}$

Well Bore Position Uncertainty: In 3D, the confidence region is most often depicted as ellipsoid because ellipsoids are the constant value contours of the 3D Guassian ${ }^{2}$ probability density function. The technique used is based on the generalized linear regression model: $\vec{y}=X \vec{\beta}+\vec{\varepsilon}$; where: $\vec{y}$ is an $(m)$ by one vector of observations. $\vec{\beta}$ is a $(p)$ by one vector of model parameters. $\mathrm{X}$ is an $(m)$ by $(p)$ matrix of regression variables, which establishes a linear relationship between the observations and the model parameters. $\overrightarrow{\mathcal{E}}$ is an $(m)$ by one vector of random errors that characterizes the uncertainty observation. $(m)$ is the number of columns in the vector $\vec{y}$. (n) is the north component of a position vector. $(p)$ is the probability density. Assuming $\overrightarrow{\mathcal{E}}$ is zero mean and has a Gaussion probability distribution, the probability density function for the random variable $\vec{y}-X \vec{\beta}$ is:

$p(\vec{y} ; \vec{\beta})=\frac{\exp \left[-\frac{1}{2}(\vec{y}-X \vec{\beta})^{T} C_{\vec{\varepsilon}}^{-1}(\vec{y}-X \vec{\beta})\right]}{2 \pi^{m / 2} \sqrt{\operatorname{det}\left(C_{\vec{\varepsilon}}\right)}}$

where: $C_{\vec{\varepsilon}}$ is the covariance matrix for the random vector $\overrightarrow{\mathcal{E}}$. Maximization of Eq.4 with respect to $\vec{\beta}$ yields the following estimate $\hat{\vec{\beta}}$ and its covariance $C_{\vec{\beta}}$. $\hat{\vec{\beta}}=\left(X^{T} C_{\vec{\varepsilon}}^{-1} X\right)^{-1} X^{T} C_{\vec{\varepsilon}}^{-1} \vec{y}$

$C_{\hat{\hat{\beta}}}=\left(X^{T} C_{\vec{\varepsilon}}^{-1} X\right)^{-1}$

$\mathrm{X}^{\mathrm{T}}$ is the transpose of $\mathrm{X}$. Assume we have $(k)$ measurement can be written in the following form: $\vec{r}_{i}=\vec{r}_{t}+\delta \vec{r}_{i}$ the position vector of the ith measurement is: $\vec{r}_{i}=\left[\begin{array}{l}n_{i} \\ e_{i} \\ d_{i}\end{array}\right]$, the true position vector is: $\vec{r}_{t}=\left[\begin{array}{l}n_{t} \\ e_{t} \\ d_{t}\end{array}\right]$ and the error in the ith measurement is: $\delta \vec{r}_{i j}=\left[\begin{array}{c}\delta n_{i j} \\ \delta e_{i j} \\ \delta d_{i j}\end{array}\right]$

A sequence of these position measurements can be written in the following form: $\left[\begin{array}{l}\vec{r}_{1} \\ \vec{r}_{2} \\ \vdots \\ \vec{r}_{k}\end{array}\right]=\left[\begin{array}{l}I_{1} \\ I_{2} \\ \vdots \\ I_{k}\end{array}\right] \vec{r}_{i}+\left[\begin{array}{l}\delta \vec{r}_{1} \\ \delta \vec{r}_{2} \\ \vdots \\ \delta \vec{r}_{k}\end{array}\right]$ in which each $\mathrm{I}_{\mathrm{j}}$ is a $(3 * 3)$ identity matrix and $1 \leq \mathrm{j} \leq \mathrm{k}$. The covariance matrix, $C_{\vec{\varepsilon}}$, can be written as:

$C_{\vec{\varepsilon}}=\left\langle\vec{\varepsilon} \vec{\varepsilon}^{T}\right\rangle=\left(\begin{array}{cccc}\left\langle\delta \vec{r}_{1} \delta \vec{r}_{1}^{T}\right\rangle & \left\langle\delta \vec{r}_{1} \delta \vec{r}_{2}^{T}\right\rangle & \ldots & \left\langle\delta \vec{r}_{1} \delta \vec{r}_{k}^{T}\right\rangle \\ \left\langle\delta \vec{r}_{2} \delta \vec{r}_{1}^{T}\right\rangle & \left\langle\delta \vec{r}_{2} \delta \vec{r}_{2}^{T}\right\rangle & \ldots & \left\langle\delta \vec{r}_{2} \delta \vec{r}_{k}^{T}\right\rangle \\ \langle\vdots\rangle & \langle\vdots\rangle & \ddots & \langle\vdots\rangle \\ \left\langle\delta \vec{r}_{k} \delta \vec{r}_{1}^{T}\right\rangle & \left\langle\delta \vec{r}_{k} \delta \vec{r}_{2}^{T}\right\rangle & \ldots & \left\langle\delta \vec{r}_{k} \delta \vec{r}_{k}^{T}\right\rangle\end{array}\right)$

where, $(d)$ is the vertical component of a position vector. $(e)$ is the east component of a position vector. $(i)$ is an integer between 1 and $\mathrm{k}$ that designate the ith member of a set of $(k)$ measurement. $(j)$ is an integer between 1 and $\mathrm{k}$ that designate the ith member of a set of $\mathrm{k}$ measurements. $(k)$ is the number of position measurements included in the ith estimate. $(t)$ is a tag used to designate the true bottom hole location. $\vec{r}_{i}$ is the ith measurement of position vector. $\vec{r}_{t}$ is the true position vector. $\delta \vec{r}_{i j}$ is the uncertainty in the ith 
measured position vector. Each term of the form $\left\langle\delta \vec{r}_{i j} \delta \vec{r}_{i j}^{T}\right\rangle$ is a $\left(3^{*} 3\right)$ covariance matrix defines a $3 \mathrm{D}$ Guassion distribution with a probability density function in the following form:

$p\left(\vec{r}_{i}\right)=\frac{\exp \left[\frac{-1}{2}\left(\vec{r}_{i}-\vec{r}_{t}\right)^{T} C_{i i}^{-1}\left(\vec{r}_{i}-\vec{r}_{t}\right)\right]}{2 \pi^{3 / 2} \sqrt{\operatorname{det}\left(C_{i i}\right)}}$

and because the covariance matrices, $\mathrm{C}_{\mathrm{ii}}$, are diagonal, the probability density function reduces to:

$$
p\left(\vec{r}_{i}\right)=\frac{\exp \left[\frac{-1}{2}\left\{\frac{\left(x_{i}-x_{t}\right)^{2}}{C_{i i_{x}}}+\frac{\left(y_{i}-y_{t}\right)^{2}}{C_{i i_{y}}}+\frac{\left(z_{i}-z_{t}\right)^{2}}{C_{i i_{i}}}\right\}\right]}{2 \pi^{3 / 2} \sqrt{\operatorname{det}\left(C_{i i}\right)}}
$$

where, $(x)$ is the element of the position covariance of matrix in the x-coordinates. $(y)$ is the element of the position covariance of matrix in the $y$-coordinates. $(z)$ is the element of the position covariance of matrix in the Z-coordinates. The constant value contours of Eq.5 are family of ellipsoids defined by the equation of the quadratic expression in the exponent to a constant. For each ellipsoid, the length of the north, east and down semi-major axes are: $s \cdot \sqrt{C_{i i_{x}}} \quad s \cdot \sqrt{C_{i i_{y}}} \quad s \cdot \sqrt{C_{i i_{z}}}$

where, $(s)$ is the normalized length of the semi major principal axes of the confidence region ellipsoid.

The mathematical basis of the HDC technique can be summarized by restating the basic formula in the following format:

$$
\left.H D C=\left[I_{n}^{T}\left(C_{\vec{\varepsilon}}\right)_{n}^{-1} I_{n}\right)^{-1} I_{n}^{T}\left(C_{\vec{\varepsilon}}\right)_{n}^{-1}\right] \times \vec{y}
$$

The covariance matrix of the HDC is given as:

$$
C_{H D C}=\left(I_{n}^{T} C_{\vec{\varepsilon}}^{-1} I_{n}\right)^{-1}
$$

Error Accuracy Prediction: The central limit theorem ${ }^{1}$ ensures that the statistical distribution of each $\delta \hat{\vec{r}}_{t}$ will be approximately Guassion and independent of the distribution of the individual error budget, Fig. 2 and 3. The following assumptions are implicit in the error models and mathematics presented:

* Errors in calculated well position are caused exclusively by the presence of measurement errors of well bore survey station.

* Wellbore survey station are three element measurement vectors, the elements being a longhole depth $(D)$, inclination $(I)$ and azimuth $(A)$. The propagation mathematics also requires a tool angle $(\alpha)$ at each station.

* Errors from different error sources are statistically independent.
* There is a linear relationship between the size of each measurement error and the corresponding change in calculated well position.

* The combined effect on calculated well position of any number of survey stations is equal to the vector sum of their individual effects.

* No restrictive assumptions are made about the statistical distribution of measurement errors.

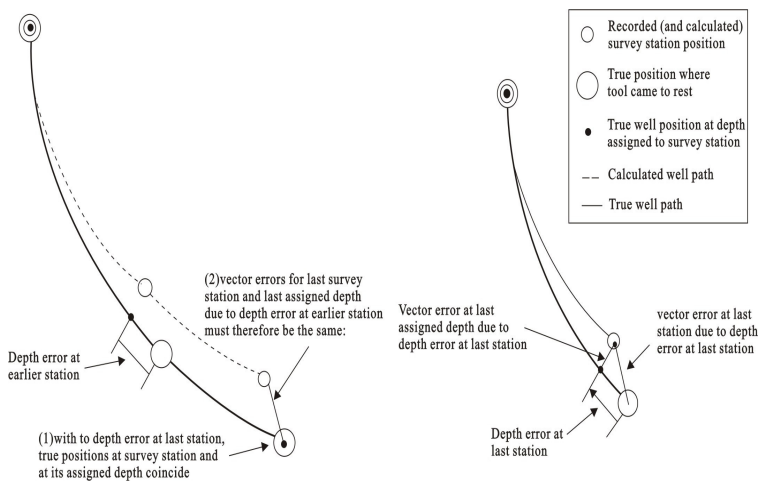

Fig. 2: Vector Error at Point of Interest

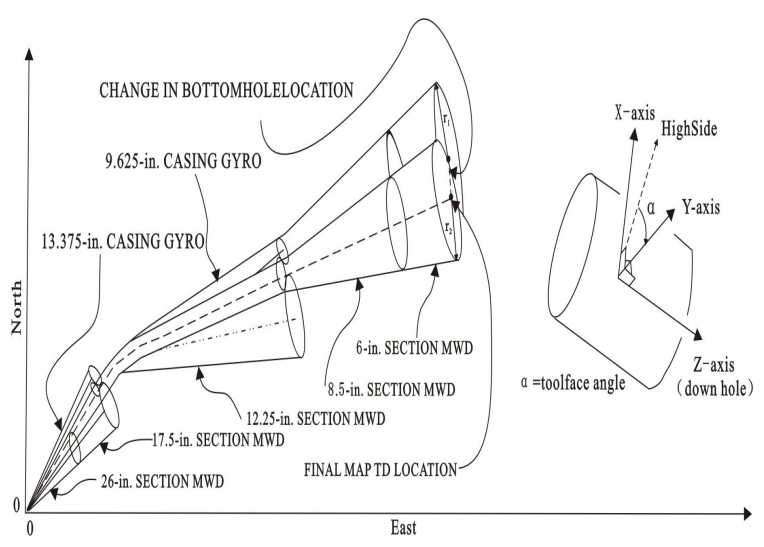

Fig. 3: The Final Section of the Well Showing Planned/Actual Wellbore Position and the Tool Face Angle Error

or the best estimate of position uncertainty it is temping to differentiate minutely among tools type and models, summing configurations, bottom hole assembly (BHA) design, geographical location and several other variables. While justifiable on technical ground, such an approach is impractical for the daily work of the well planner.

The Error Propagation Mathematical Model: The method of position uncertainty calculation admits a number of variations, in that selection of the same set of conventions which always yield the same results. Recall and evaluate the vector error due to the presence of 
error source (i) at the station $\mathrm{k}$, which is the sum of the effect of the error on the preceding and following survey displacement yield:

$\Delta r_{j}=\frac{D_{j}-D_{j-1}}{2}\left[\begin{array}{c}\sin I_{j-1} \cos A_{j-1}+\sin I_{j} \cos A_{j} \\ \sin I_{j-1} \sin A_{j-1}+\sin I_{j} \sin A_{j} \\ \cos I_{j-1}+\cos I_{j}\end{array}\right]$

the two differentials in the parentheses in Eq.8 may then be expressed as:

$$
\begin{aligned}
& \frac{d \Delta r_{k}}{d p_{k}}=\left[\frac{d \Delta r_{j}}{d D_{k}}+\frac{d \Delta r_{j}}{d I_{k}}+\frac{d \Delta r_{j}}{d A_{k}}\right] \\
& \frac{d \Delta r_{k}}{d D_{k}}=\frac{1}{2}\left[\begin{array}{c}
\sin I_{k-1} \cos A_{k-1}+\sin I_{k} \cos A_{k} \\
\sin I_{k-1} \sin A_{k-1}+\sin I_{k} \sin A_{k} \\
\cos A_{k-1}+\cos A_{k}
\end{array}\right] \\
& \left.\frac{d \Delta r_{k+1}}{d D_{k}}=\frac{1}{2}\left[\begin{array}{c}
-\sin I_{k} \cos A_{k}+\sin I_{k+1} \cos A_{k+1} \\
-\sin I_{k} \sin A_{k}+\sin I_{k+1} \sin A_{k+1} \\
-\cos A_{k}+\cos A_{k+1}
\end{array}\right]\right] \\
& \frac{d \Delta r_{j}}{d I_{k}}=\frac{1}{2}\left[\begin{array}{c}
\left(D_{j}-D_{j-1}\right) \cos I_{k} \cos A_{k} \\
\left(D_{j}-D_{j-1}\right) \cos I_{k} \cos A_{k} \\
\left(D_{j}-D_{j-1}\right) \sin I_{k}
\end{array}\right] \\
& \frac{d \Delta r_{j}}{d A_{k}}=\frac{1}{2}\left[\begin{array}{l}
-\left(D_{j}-D_{j-1}\right) \sin I_{k} \sin A_{k} \\
-\left(D_{j}-D_{j-1}\right) \sin I_{k} \sin A_{k}
\end{array}\right]
\end{aligned}
$$

for the purpose of computation the error summation terminated at the survey station of interest the vector errors at this station are therefore given by:

$$
e_{i, l, k}^{*}=\sigma_{i, l} \bullet \frac{d \Delta r_{k}}{d p_{k}} \bullet \frac{\partial p_{k}}{\partial \varepsilon_{i}}
$$

where, $\left(e^{*}\right)$ is the 1s.d vector error of the station of interest.

Writing $\Delta r_{k}$ for the displacement between survey station (k-1) and (k), it may express the 1s.d error due to the presence of the ith error at the kth survey station in the lth survey leg as the sum of the effect on preceding and following calculated displacement.

$$
e_{i, l, k}=\sigma_{i, l}\left(\frac{d \Delta r_{k}}{d p_{k}}+\frac{d \Delta r_{k+1}}{d p_{k+1}}\right) \frac{\partial p_{k}}{\partial \varepsilon_{i}}
$$

where: $(e)$ is the 1s.d vector error at an intermediate station. $\sigma$ is the standard deviation of error vector. $(r)$ is the wellbore position vector. $(p)$ is the survey measurement vector $(D, I, A), \mathcal{E}$ is the particular value of a survey error. $\partial p_{k} / \partial \varepsilon_{i}$ describes how is the changes in the measurement vector affect the calculated well position.

Weighting Functions for Sensor Errors: The weighting functions for constant and $\mathrm{B}_{\mathrm{H}}$-dependent magnetic declination errors are:

$\frac{\partial p}{\partial \varepsilon_{A Z}}=\left[\begin{array}{l}0 \\ 0 \\ 1\end{array}\right] \quad \frac{\partial p}{\partial \varepsilon_{D B_{H}}}=\left[\begin{array}{c}0 \\ 0 \\ 1 / B \cos \Theta\end{array}\right]$

for BHA sag and direction-dependent axial magnetic interference they are:

$\frac{\partial p}{\partial \varepsilon_{s a g}}=\left[\begin{array}{c}0 \\ \sin I \\ 0\end{array}\right] \quad \frac{\partial p}{\partial \varepsilon_{A M I_{D}}}=\left[\begin{array}{c}0 \\ 0 \\ \sin I \sin A_{m}\end{array}\right]$

and for reference, scale and stretch type depth error they are:

$\frac{\partial p}{\partial \varepsilon_{D_{R E F}}}=\left[\begin{array}{l}1 \\ 0 \\ 0\end{array}\right] \frac{\partial p}{\partial \varepsilon_{D_{S F}}}=\left[\begin{array}{c}D \\ 0 \\ 0\end{array}\right] \quad \frac{\partial p}{\partial \varepsilon_{D_{S T}}}=\left[\begin{array}{c}D \cdot D_{v} \\ 0 \\ 0\end{array}\right]$

where: $(B)$ is the magnetic declination, $\mathrm{nT} . \Theta$ is the magnetic dip angle, deg.

Tool axis and tool angle are defined in Fig. 2. There are 12 sensor error sources and each requires its own weight function. These are obtained by differentiating the standard navigation equations for inclination and azimuth:

$$
I=\cos ^{-1} \frac{G}{\sqrt{G_{x}^{2}+G_{y}^{2}+G_{z}^{2}}}
$$

$A_{m}=\tan ^{-1}\left(\frac{\left(G_{x} B_{y}-G_{y} B_{x}\right) \sqrt{G_{x}^{2}+G_{y}^{2}+G_{z}^{2}}}{B_{z}\left(G_{x}^{2}+G_{y}^{2}-G_{z}\left(G_{x} B_{x}+G_{y} B_{y}\right)\right)}\right)$

and making use of the inverse relations:

$G_{x}=-G \sin I \sin \alpha$

$G_{y}=-G \sin I \cos \alpha \quad G_{z}=G \cos I$

$B_{x}=B \cos \Theta \cos I \cos A_{m} \sin \alpha-B \sin \Theta \sin I \sin \alpha+B \cos \Theta \sin A_{m} \cos \alpha$

$B_{y}=B \cos \Theta \cos I \cos A_{m} \cos \alpha-B \sin \Theta \sin I \cos \alpha-B \cos \Theta \sin A_{m} \sin \alpha$

$B_{z}=B \cos \Theta \sin I \cos A_{m}+B \sin \Theta \cos I$

Effect of Axial Interference Correction: Detailed of the interference corrections differ from method to method, but it is reasonable to characterize them all. From Eq. 15 and ignoring $\mathrm{B}_{\mathrm{z}}$ measurement; then 
$(B \cos \Theta-\hat{B} \cos \hat{\Theta})^{2}+(B \sin \Theta-\hat{B} \sin \hat{\Theta})^{2}=$ MINIMUM

where $\hat{B}$ and $\hat{\Theta}$ are the estimated values of total field strength and dip angle respectively. Solving these three equations for azimuth leads to:

$P \sin A_{m}+Q \cos A_{m}+R \sin A_{m} \cos A_{m}=0$

where, $\left.P=\left(B_{x} \sin \alpha+B_{y} \cos \alpha\right) \cos I+\hat{B} \sin \hat{\Theta} \sin I \cos I\right)$

$Q=-\left(B_{x} \cos \alpha-B_{y} \sin \alpha\right) ; R=\hat{B} \cos \hat{\Theta} \sin ^{2} I$. The sensitive of computed azimuth to error in the sensor measurement are found by differentiating Eq.16 with respect to $\hat{B}$ and $\hat{\Theta}$. The misalignment error modeled by William ${ }^{3}$ as two uncorrelated errors corresponding to the $\mathrm{X}$-axis and $\mathrm{Y}$-axis of the associated inclination and azimuth error lead directly to the following weighting function:

$$
\frac{\partial p}{\partial \varepsilon_{M X}}=\left[\begin{array}{c}
0 \\
\sin \alpha \\
-\cos \alpha / \sin I
\end{array}\right] \quad \frac{\partial p}{\partial \varepsilon_{M Y}}=\left[\begin{array}{c}
0 \\
\cos \alpha \\
\sin \alpha / \sin I
\end{array}\right]
$$

Summation of Errors: The contribution to survey station uncertainty from randomly propagation error source $(i)$ over survey leg $(l)$ (not containing the station of interest is:

$[C]_{i . l}^{\text {rand }}=\sum_{k=1}^{k_{1}}\left(e_{i, l, k}\right) \bullet\left(e_{i, l, k}\right)^{T}$

and the total contribution over all survey legs is

$[C]_{i, k}^{r a n d}=\sum_{l=1}^{L-1}[C]_{i, l}^{r a n d}+\sum_{k=1}^{k_{1}}\left(e_{i, l, k}\right) \bullet\left(e_{i, l, k}\right)^{T}+\left(e_{i, l, k}^{*}\right) \bullet\left(e_{i, l, k}^{*}\right)^{T}$

The contribution to survey station uncertainty from a systematic propagation error $(i)$ over survey leg $(l)$ is:

$[C]_{i, k}^{\mathrm{syst}}=\sum_{l=1}^{L-1}[C]_{i, k}^{\mathrm{sys} t}+\left(\sum_{k=1}^{k_{1}} e_{i, l, k}+e_{i, l, k}^{*}\right)\left(\sum_{k=1}^{k_{1}} e_{i, l, k}+e_{i, l, k}^{*}\right)^{T}$

Each of these error types is systematic among all stations in a well. The individual errors therefore are summed to give a total vector error from slot to station:

$$
E_{i, k}=\sum_{l=1}^{L-1}\left(\sum_{k=1}^{k_{1}} e_{i, l, k}\right)+\sum_{l=1}^{L-1}\left(\sum_{k=1}^{k_{1}} e_{i, l, k}^{*}\right)
$$

the total contribution to the uncertainty at survey station $\mathrm{K}$ is: $C_{i, k}^{\text {well }}=E_{i, k} \bullet E_{i, k}^{T}$

where: $(E)$ is the sum of vector errors from slot to station of interest.
The total position covariance at survey station $(K)$ is the sum of the contributions from all the types of error source:

$$
[C]_{K}^{\text {sur }}=\sum_{i \in R}[C]_{i, K}^{\mathrm{rand}}+\sum_{i \in S}[C]_{i, K}^{\mathrm{sys} t}+\sum_{i \in\{W, G\}}[C]_{i, K}^{\text {well }}
$$

where the superscript (sur) indicates the uncertainty is defined at a survey station.

Error vectors due to bias error are given by:

$$
m_{i, l, k}=\mu_{i, l}\left(\frac{d \Delta r_{k}}{d p_{k}}+\frac{d \Delta r_{k+1}}{d p_{k}}\right) \frac{\partial p_{k}}{\partial \varepsilon_{i}} \quad m_{i, l, k}^{*}=\mu_{i, L} \frac{d \Delta r_{k}}{d p_{k}} \frac{\partial p_{k}}{\partial \varepsilon_{i}}
$$

where, $(m)$ is the bias vector error at an intermediate station. $\left(m^{*}\right)$ is the bias vector error at the station of interest.

The total survey position bias at survey station $(K$, $M_{K}^{\text {sur }}$ ) is the sum of individual bias vectors taken over all error source $(i)$, legs $(l)$ and station $(k)$ :

$$
M_{K}^{s u r}=\sum_{i}\left(\sum_{l=1}^{L-1}\left(\sum_{k=1}^{K_{1}} m_{i, l, k}+\sum_{k=1}^{K-1}\left(m_{i, l, k}+m_{i, l, k}^{*}\right)\right)\right)
$$

Defining the superscript (dep) to indicate uncertainty at an assigned depth, it may be shown that:

$$
e_{i, l, k}^{* d e p}=e_{i, l, k}^{* s u r}-\sigma_{i, L} W_{i, L, K} v_{K} \quad e_{i, l, k}^{d e p}=e_{i, l, k}^{s u r}
$$

where, $\left(W_{i, L, K}\right)$ is the factor relating error magnitude to depth measurement uncertainty. $\left(v_{k}\right)$ is the along-hole unit vector at station K. Fig. 4 illustrates these results.

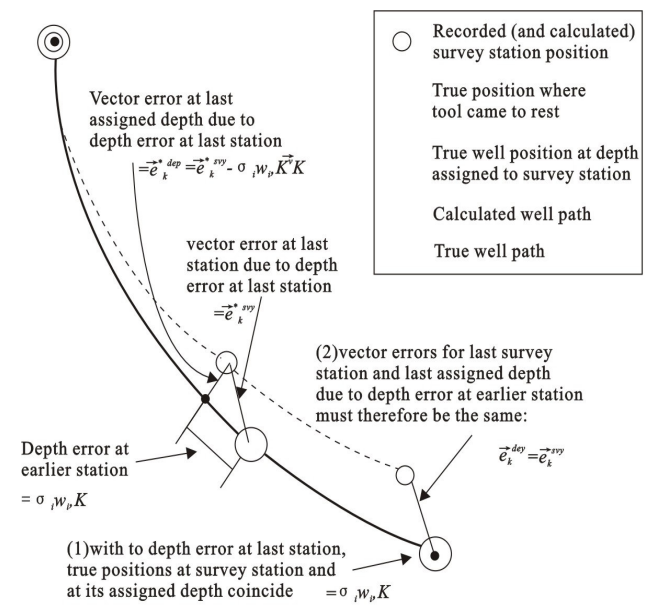

Fig. 4: Vector Errors at the Last Station

Survey bias at an assigned depth is calculated by:

$m_{i, L, K}^{* d e p}=m_{i, L, K}^{* \text { sur }}-\mu_{i, L} W_{i, L, K} v_{K} \quad m_{i, L, K}^{d e p}=m_{i, L, K}^{s u r}$ 
When calculating the uncertainty in the relative position between two surveys stations $\left(K_{A}, K_{B}\right)$, the uncertainty is given by:

$[C]^{s u r}\left[r_{K_{A}}-r_{K_{B}}\right]=[C]_{K_{A}}^{s u r}+[C]_{K_{B}}^{s u r}$

$-\sum_{i=G}\left\{\left(E_{i, K_{A}}\right) \bullet\left(E_{i, K_{B}}\right)^{T}+\left(E_{i, K_{B}}\right) \bullet\left(E_{i, K_{A}}\right)^{T}\right\}$

the relative survey bias is simply: $M^{\operatorname{sur}}\left[r_{K_{A}}-r_{K_{B}}\right]=M_{K_{A}}^{\text {sur }}-M_{K_{B}}^{\text {sur }}$

The uncertainty in this position error is expressed in the form of a covariance matrix:

$$
\begin{aligned}
& {\left[C_{K}\right]=\left\langle\delta \vec{r}_{i j} \bullet \delta \vec{r}_{i j}^{T}\right\rangle=} \\
& \sum_{\text {errors }(\mathrm{i}, \mathrm{j})} \sum_{\mathrm{K}_{\mathrm{i}} \leq K} \sum_{K_{j} \leq K}\left\{\begin{array}{l}
\rho\left(\varepsilon_{i, l_{i i}, k_{i i}} ; \varepsilon_{i, l_{j j}, k_{j j}}\right) e_{i, l_{i i}, k_{i i}} \cdot e_{i, l_{j j}, k_{j j}}^{T} \\
+\rho\left(\varepsilon_{i, l_{j j}, k_{j j}} ; \varepsilon_{i, l_{i i}, k_{i i}}\right) e_{i, l_{j j}, k_{j j}} \cdot e_{i, l_{i i}, k_{i i}}^{T}
\end{array}\right\}
\end{aligned}
$$

The results derived above are in an Earth-Referenced frame (north, east, vertical, subscript (nev)). The transformation of the covariance matrices and bias vector into the more intuitive borehole referenced frame (high side, lateral hole, subscript $(h l a)$ ) is straightforward: $[C]_{h l a}=[T] \bullet[T]^{T}\left[C \bullet C^{*}\right]_{n e v}$

$\left[\begin{array}{c}b_{H} \\ b_{L} \\ b_{A}\end{array}\right]=[T]^{T} M_{\text {nev }}[T]=\left[\begin{array}{ccc}\cos I_{K} \cos A_{K} & -\sin A_{K} & \sin I_{K} \cos A_{K} \\ \cos I_{K} \sin A_{K} & \cos A_{K} & \sin I_{K} \sin A_{K} \\ -\sin I_{K} & 0 & \cos I_{K}\end{array}\right]$

[T] is a rotation matrix. The uncertainties and correlations in the principal borehole directions are obtained from:

$\sigma_{H}=\frac{\sqrt{[C]_{h l a}[I, I]}}{[I, J]}$ etc. $\rho_{H A}=\frac{[C]_{\text {hla }}[I, G]}{\sigma_{H} \sigma_{L}}$ etc.

\section{RESULTS AND CONCLUSION}

The error models for basic interference-correction MWD have been applied to the standard well profiles to generate position uncertainties in each well. The results of several combinations are tabulated in Table 1 and 2.

Table 1: Standard Well Profile

Well 1: lat. $=60^{\circ} \mathrm{N}, \log .=2^{\circ} \mathrm{E}, \mathrm{G}=9.80665 \mathrm{~m} \mathrm{~s}^{-2}, \mathrm{~B}=50000 \mathrm{nT}, \Theta=72^{\circ}, \delta=4^{\circ} \mathrm{W}$, station interval $=30 \mathrm{~m}$, vertical section azimuth $=75^{\circ}$

\begin{tabular}{lccccccc} 
MD $(\mathrm{m})$ & Inc $(\mathrm{deg})$ & Azi $(\mathrm{deg})$ & North $(\mathrm{m})$ & East $(\mathrm{m})$ & TVD $(\mathrm{m})$ & VS $(\mathrm{m})$ & DLS $\% / 30 \mathrm{~m}$ \\
\hline 0 & 0 & 0 & 0 & 0 & 0 & 0 & 0 \\
1200 & 0 & 0 & 0 & 0 & 1200 & 0 & 0 \\
2100 & 60 & 75 & 111.22 & 415.08 & 1944.29 & 429.79 & 2 \\
5100 & 60 & 75 & 783.65 & 2924.62 & 3444.29 & 3027.79 & 0 \\
5400 & 90 & 75 & 857.8 & 3201.34 & 3521.06 & 3314.27 & 3 \\
5850 & 90 & 75 & 1530.73 & 5712.75 & 3521.06 & 5914.27 & 0 \\
\hline
\end{tabular}

Well 2: lat. $=28^{\circ} \mathrm{N}, \log .=90^{\circ} \mathrm{E}, \mathrm{G}=9.80665 \mathrm{~m} \mathrm{~s}^{-2}, \mathrm{~B}=48000 \mathrm{nT}, \Theta=58^{\circ}, \delta=2^{\circ} \mathrm{E}$, station interval $=100 \mathrm{~m}$, vertical section azimuth $=21^{\circ}$

\begin{tabular}{lccccccc}
0 & 0 & 0 & 0 & 0 & 0 & 0 & 0 \\
609.6 & 0 & 0 & 0 & 0 & 609.6 & 0 & 0 \\
1079.28 & 32 & 2 & 435.4 & 15.19 & 1072.32 & 411 & 2 \\
1524 & 32 & 2 & 1176.48 & 41.08 & 1434.2 & 1113.06 & 0 \\
1684.185 & 32 & 32 & 1435.37 & 20.23 & 1570.91 & 1383.12 & 3 \\
1844.37 & 32 & 62 & 1619.99 & 318.22 & 1707.615 & 1626.43 & 3 \\
2004.554 & 32 & 92 & 1680.89 & 582 & 2013.232 & 1777.82 & 3 \\
2164.74 & 32 & 122 & 1601.74 & 840.88 & 2062.057 & 1796.7 & 3 \\
2862.0263 & 62 & 220 & 364.88 & 700 & 2519.254 & 591.63 & 3 \\
3810 & 62 & 220 & -1692.7 & -1026.15 & 2991.923 & -1948.01 & 0 \\
\hline
\end{tabular}

Well 3: lat. $=40^{\circ} \mathrm{S}, \log .=147^{\circ} \mathrm{E}, \mathrm{G}=9.80665 \mathrm{~m} \mathrm{~s}^{-2}, \mathrm{~B}=61000 \mathrm{nT}, \Theta=-70^{\circ}, \delta=13^{\circ} \mathrm{E}$, station interval $=30 \mathrm{~m}$,

\begin{tabular}{|c|c|c|c|c|c|c|c|}
\hline 0 & 0 & 0 & 0 & 0 & 0 & 0 & 0 \\
\hline 500 & 0 & 0 & 0 & 0 & 500 & 0 & 0 \\
\hline 1100 & 50 & 0 & 245.6 & 0 & 1026.69 & 198.7 & 2.5 \\
\hline 1700 & 50 & 0 & 705.23 & 0 & 1412.37 & 570.54 & 0 \\
\hline 2450 & 0 & 0 & 1012.23 & 0 & 2070.73 & 818.91 & 2 \\
\hline 2850 & 0 & 0 & 1012.23 & 0 & 2470.73 & 818.91 & 0 \\
\hline 3030 & 90 & 283 & 1038.01 & -111.65 & 2585.32 & 905.39 & 15 \\
\hline 3430 & 90 & 283 & 1127.99 & -501.4 & 2585.32 & 1207.28 & 0 \\
\hline 3730 & 110 & 193 & 996.08 & -727.87 & 2520 & 1197.85 & 9 \\
\hline 4030 & 110 & 193 & 721.4 & -791.28 & 2417.4 & 1069.86 & 0 \\
\hline
\end{tabular}
vertical section azimuth $=310^{\circ}$ 
Table 2: Calculated position uncertainties (1s.d)

Uncertainties A Long-Borehole Axes

\begin{tabular}{rllllrrr}
\hline & Well No. & Depth interval $(\mathrm{m})$ & Model & Option & $\sigma_{\mathrm{H}}(\mathrm{m})$ & $\sigma_{\mathrm{L}}(\mathrm{m})$ & \multicolumn{1}{c}{$\sigma_{\mathrm{A}}(\mathrm{m})$} \\
\hline 1 & 1 & 0 to 2500 & Basic & S, sym & 20.116 & 84.342 & 8.626 \\
2 & 1 & 0 to 2500 & Ax-int & S, sym & 20.116 & 196.390 & 8.626 \\
3 & 2 & 0 to 3800 & Basic & S, sym & 16.185 & 29.551 & 10.057 \\
4 & 2 & 0 to 3800 & Basic & D, sym & 16.185 & 29.551 & 9.080 \\
5 & 2 & 0 to 3800 & Basic & S, bias & 15.710 & 27.288 & 8.526 \\
6 & 2 & 0 to 3800 & Basic & D, bias & 15.710 & 27.288 & 8.419 \\
7 & 3 & (1) 0 to 1380 & Basic & S, sym & 2.013 & 3.703 & 0.919 \\
& (2) 1410 to 3000 & Ax-ani & S, sym & 3.239 & 3.646 & 7.890 \\
& (3) 3030 to 4030 & basic & S, sym & 5.604 & 9.594 & 9.594 \\
\hline
\end{tabular}

Correlation Between Borehole Axes

\begin{tabular}{|c|c|c|c|c|c|c|c|}
\hline & Well No. & Depth interval $(\mathrm{m})$ & Model & Option & $\rho_{H L}$ & $\rho_{H \Lambda}$ & $\rho_{L A}$ \\
\hline 1 & 1 & 0 to 2500 & Basic & S, sym & -0.016 & +0.676 & -0.004 \\
\hline 2 & 1 & 0 to 2500 & Ax-int & $\mathrm{S}$, sym & -0.005 & +0.676 & -0.005 \\
\hline 3 & 2 & 0 to 3800 & Basic & S, sym & +0.030 & -0.613 & +0.049 \\
\hline 4 & 2 & 0 to 3800 & Basic & $\mathrm{D}$, sym & +0.030 & -0.429 & +0.073 \\
\hline 5 & 2 & 0 to 3500 & Basic & $\mathrm{S}$, bias & +0.050 & -0.607 & +0.145 \\
\hline 6 & 2 & 0 to 3800 & Basic & $\mathrm{D}$, bias & +0.050 & -0.574 & +0.148 \\
\hline \multirow[t]{3}{*}{7} & 3 & (1) 0 to 1380 & Basic & S, sym & -0.007 & 0.633 & -.006 \\
\hline & & (2) 1410 to 3000 & Ax-ani & $\mathrm{S}$, sym & -0.172 & 0.633 & -0.665 \\
\hline & & (3) 3030 to 4030 & basic & S, sym & -0.180 & -0.590 & +0.302 \\
\hline
\end{tabular}

Survey Bias A Long- Borehole Axis

\begin{tabular}{|c|c|c|c|c|c|c|c|}
\hline & Well No. & Depth interval $(\mathrm{m})$ & Model & option & $b_{H}(\mathrm{~m})$ & $b_{L}(\mathrm{~m})$ & $b_{A}(\mathrm{~m})$ \\
\hline 1 & 1 & 0 to 2500 & Basic & S, sym & & & \\
\hline 2 & 1 & 0 to 2500 & Ax-int & S, sym & & & \\
\hline 3 & 2 & 0 to 3800 & Basic & S, sym & & & \\
\hline 4 & 2 & 0 to 3800 & Basic & $\mathrm{D}$, sym & & & \\
\hline 5 & 2 & 0 to 3800 & Basic & $\mathrm{S}$, bias & -6.788 & -12.4117 & +11.698 \\
\hline 6 & 2 & 0 to 3800 & Basic & $\mathrm{D}$, bias & -6.788 & -12.411 & -4.758 \\
\hline \multirow[t]{3}{*}{7} & 3 & (1) 0 to 1380 & Basic & S, sym & & Results at & \\
\hline & & (2) 1410 to 3000 & Ax-int & S, sym & & Results at & \\
\hline & & (3) 3030 to 4030 & basic & S, sym & & Results at & \\
\hline \multirow{2}{*}{\multicolumn{3}{|c|}{ Key to error model }} & basic & & C MWD & & \\
\hline & & & Ax-int & \multicolumn{4}{|l|}{ Basic 1} \\
\hline \multirow{4}{*}{\multicolumn{3}{|c|}{ Key to calculation options }} & S, sym & \multicolumn{4}{|c|}{$\begin{array}{l}\text { Uncertainty at survey station, all errors symmetric (i.e., no } \\
\text { bias). }\end{array}$} \\
\hline & & & $\mathrm{S}$, bias & \multicolumn{4}{|c|}{$\begin{array}{l}\text { Uncertainty at survey station, selected errors symmetric } \\
\text { modeled as bias. }\end{array}$} \\
\hline & & & D, sym & \multirow{2}{*}{\multicolumn{4}{|c|}{$\begin{array}{l}\text { Uncertainty at assigned depth, all errors symmetric (i.e., no bias) } \\
\text { Uncertainty at assigned depth, selected errors symmetric } \\
\text { modeled as bias. }\end{array}$}} \\
\hline & & & $\mathrm{D}$, bias & & & & \\
\hline
\end{tabular}

Uncertainties at the tie line $(\mathrm{MD}=0)$ is zero; stations interpolated at whole multiples of station interval using minimum curvature and minimum distance methods; well plan way points included as additional stations; instrument tool face $=$ borehole tool face

Example 1 and 2 (Table 2) compare the basic and interference in well Unity\#30. Being a high inclination well running an approximately, the interference correction actually degrades the accuracy. The results are plotted in Fig. 5. Example 3 to 6 all represent the basic MWD error model applied to well RenMen\#95.
They differ in that each uses a different permutation of the survey station/assigned depth and symmetric error/survey bias calculation options. The variation of lateral uncertainty and ellipsoid semi-major axis, characteristics is shown in Fig. 6. 


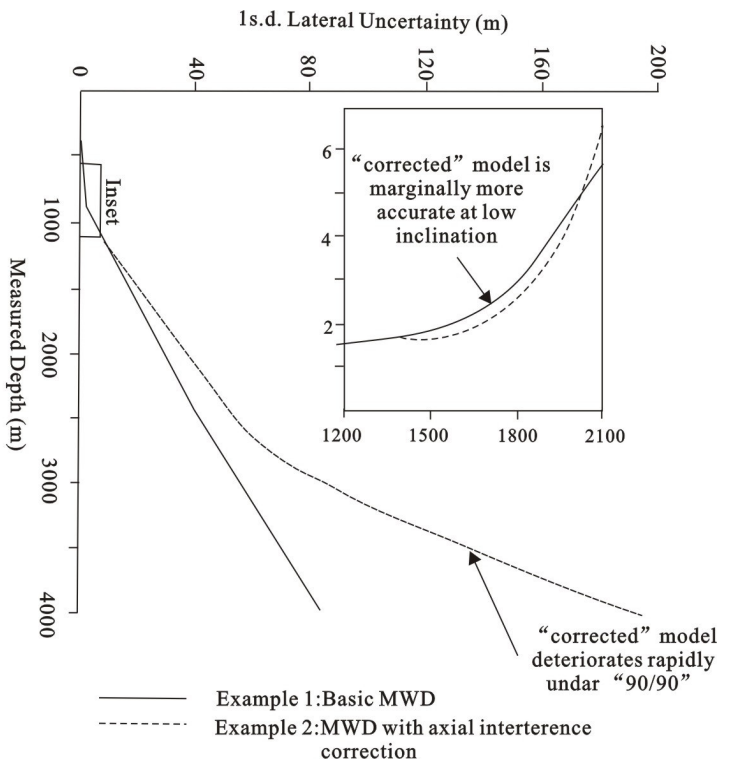

Fig. 5: Comparison of Basic and Interference Corrected MWD Error Models Well Unity\#30

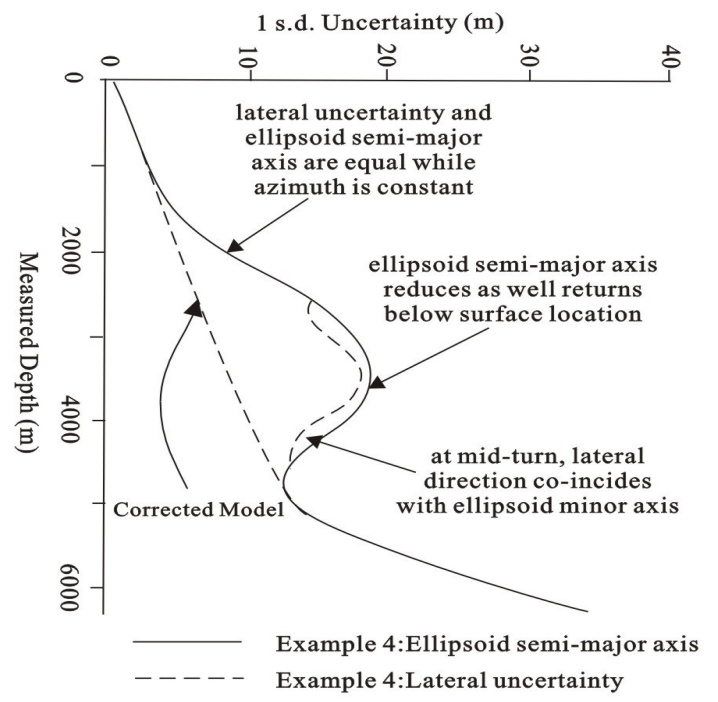

Fig. 6: Variation of lateral uncertainty and ellipsoid semi-major axis well RenMen\#95

Example 7 breaks well Quan\#95 into three depths intervals, with the basic and interference-correction models being applied alternately. This example is included as a test of error propagation (Fig. 7 and 8).

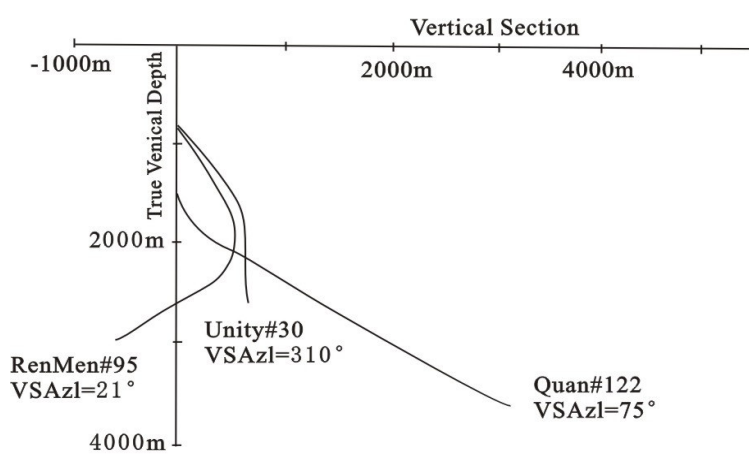

Fig. 7: Vertical Section of Well Profiles

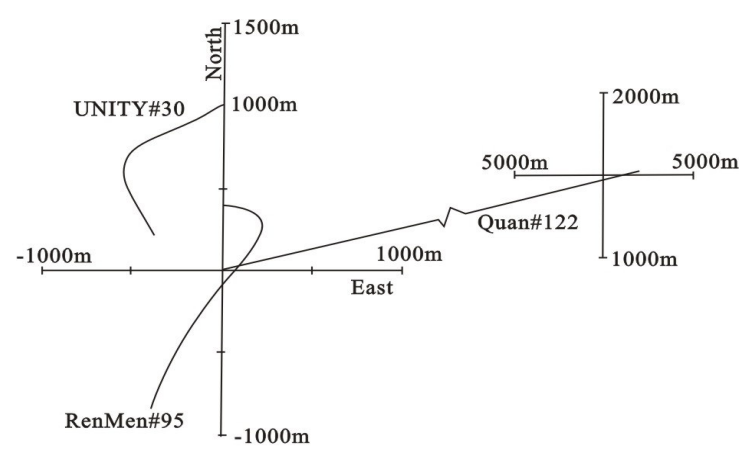

Fig. 8: Plan View of Well Profile

\section{REFERENCES}

1. Ekseth, R., 1998. Uncertainties in connection with the determination of wellbore position. Ph.D. Thesis. Norwegian University of Science and Technology. Trondheim, Norway.

2. Kay, S.M., 1992. Fundamental of Statistical Signal Processing. Prentice Hall, Englewood Cliffs, New Jersey, pp: 141.

3. Williamson, H.S., 1999. Accuracy prediction for directional measurement while drilling. SPE-67616 First Presented in the 1999 SPE Annual Technical Conference and Exhibition, Houston, 3-6 Oct. 